\title{
PERCEPÇÃO DOS ENFERMEIROS SOBRE ESTRATIFICAÇÃO DE RISCO EM SAÚDE MENTAL E AS AÇÕES DE ENFERMAGEM
}

\author{
Nurses' perception of the stratification of risk in \\ mental health and the nursing actions
}

Fernanda Pedersoli Lopes ${ }^{1}$, Marcelle Paiano ${ }^{2}$, Maria Emília Grassi Busto Miguel ${ }^{3}$, Maria Aparecida Salci ${ }^{4}$

1. Enfermeira. Graduada pela Universidade Estadual de Maringá (UEM). ORCID: https://orcid.org/00000002-6461-6037

2. Enfermeira. Doutora. Docente do Departamento de Enfermagem da UEM. ORCID: https://orcid. org/0000-0002-7597-784X

3. Enfermeira. Doutora. Docente do Departamento de Enfermagem da UEM. ORCID: https://orcid. org/0000-0002-2046-7009

4. Enfermeira. Doutora. Docente do Departamento de Enfermagem da UEM. ORCID: https://orcid. org/0000-0002-6386-1962

CONTATO: Marcelle Paiano | Endereço: Av. Colombo, n. 5790 | CEP: 87020-900 | Telefone: (44) 33014514 | E-mail: mpaiano@uem.br

COMO CITAR: Lopes FP, Paiano M, Miguel MEGB, Salci MA. Percepção dos enfermeiros sobre estratificação de risco em saúde mental e as ações de enfermagem. R. Saúde Públ. 2019 Jul;2(1):68-79.

RESUMO O presente estudo objetivou conhecer a percepção do enfermeiro sobre o instrumento de estratificação de risco em saúde mental e quais ações de enfermagem são ofertadas ao paciente classificado como baixo risco. Trata-se de estudo qualitativo, descritivo-exploratório, realizado em quatorze Unidades Básicas de municípios do Noroeste do Paraná. Para a coleta de dados, utilizou-se entrevista aberta, com vinte e cinco entrevistados. Foram elencadas três categorias: Ações em saúde mental realizadas pelos enfermeiros da ESF; Instrumento de estratificação 
de risco em saúde mental: potencialidades e fragilidades e Dificuldades das Equipes da ESF em saúde mental. Conclui-se que as ações desenvolvidas envolvem grupos de escuta e acolhimento, convivência, atividades de leitura e apoio matricial do NASF, e como fragilidades destaca-se a dificuldade da aplicação do instrumento de estratificação de risco, excesso de encaminhamentos para especialistas e falta de tempo e afinidade em saúde mental.

PALAVRAS-ChAVE: Gestão da Qualidade. Estratégia de Saúde da Família. Atenção Primária à Saúde. Saúde Mental. Gestão de Riscos. Enfermagem.

ABSTRACT The present study aimed to know the nurses' perception of the risk stratification instrument in mental health and which nursing actions are offered to the patient classified as low risk. It is a qualitative, descriptive-exploratory study, carried out in fourteen Basic Units, in the municipality of the Northwest of Paraná. For data collection, an open interview was used, with twenty-five interviewees. Three categories were listed: Mental health actions performed by the Family Health Strategy (ESF) nurses; Mental health risk stratification instrument: strengths and weaknesses, and difficulties ESF teams have when dealing with mental health. It is concluded that the actions developed involve listening and embracement groups, coexistence, reading activities, and support from the Family Health Support Center (NASF), and the weaknesses include the difficulty of applying the risk stratification instrument, excessive referrals to specialists, and lack of time and affinity in mental health.

KEYWORDS: Quality management. Family Health Strategy. Primary Health Care. Mental Health. Risk Management. Nursing.

\section{INTRODUÇÃO}

A

Saúde Mental na atenção primária é um tema complexo, no entanto, encontra-se presente no cotidiano efetivo de trabalho dos profissionais desta esfera de atendimento. As pessoas acometidas por sofrimento e/ou transtorno mental, decorrentes ou não do uso de substâncias psicoativas, desde alterações leves até as mais graves, merecem cuidado na Atenção Primária à Saúde (APS) como as demais condições crônicas de saúde 1 .
Entende-se que o meio privilegiado para tratamento das pessoas em sofrimento mental é o território, junto com suas famílias e a comunidade onde as pessoas residem. Isso ocorre porque o hospital psiquiátrico deixou de ser o foco da assistência da organização das políticas e da formação profissional, da mesma maneira que não são considerados eficientes para a recuperação das pessoas em grave sofrimento psíquico?. 
Sendo assim, muitos são os benefícios de integrar a saúde mental à APS. Algumas das vantagens principais são que a integração faz com que os indivíduos tenham acesso a serviços de saúde mental mais perto de seu domicílio, o que os mantêm junto das suas famílias e de suas atividades diárias. Os serviços de APS também facilitam as iniciativas comunitárias junto à população e a promoção da saúde mental, assim como o monitoramento e a gestão em longo prazo dos indivíduos afetados?

O cuidado da saúde mental na APS também elimina o risco das violações dos direitos humanos que ocorrem em hospitais psiquiátricos além de serem viáveis financeiramente, tanto para os usuários, quanto para as comunidades e os governos. Além disso, os usuários e suas famílias evitam os custos indiretos associados à procura de cuidados especializados em localizações distantes ${ }^{3}$.

Neste sentido, para direcionar as ações de saúde mental, foi publicado o Decreto Presidencial $n^{0} 7.508 / 2011$, que regulamenta a Lei $n^{\circ}$ 8.080/1990, que dispõe sobre a organização do SUS, o planejamento da saúde, a assistência à saúde e a articulação interfederativa, preconizando que a atenção psicossocial deve ser "uma rede indispensável nas regiões de saúde". Para sua operacionalização, seguindo as diretrizes do Sistema Único de Saúde (SUS) e da Política Nacional de Saúde Mental, foi promulgada a Portaria $n^{\circ}$ 3.088/2011 que instituiu a Rede de Atenção Psicossocial (RAPS) para pessoas em sofrimento ou transtorno mental e com necessidades decorrentes do uso de crack, álcool e outras drogas'.

Considerando que os serviços assistenciais são de responsabilidade do município, cabe ao Estado, em seu papel regulador, estimular a criação de políticas municipais em consonância com a Reforma Psiquiátrica Brasileira, articular as negociações regionalizadas, fiscalizar e oferecer suporte técnico às equipes. Assim, conforme as diretrizes e os objetivos da RAPS, as ações de saúde mental devem ser estruturadas a partir da realidade municipal/ microrregional / regional / macrorregional, considerando as necessidades e os recursos?.

Deste modo, para a efetivação da Política Estadual de Saúde Mental, a Secretaria Estadual de Saúde (SESA) do Paraná redefiniu as diretrizes e estratégias por meio de um processo de Planejamento Estratégico, considerando a realidade do Estado e procurando abranger o que a Política Nacional não contempla. Neste planejamento, a estratificação de risco da população é implantada, considerando a gravidade dos sinais e sintomas apresentados, sem a necessidade de firmar diagnóstico inicial, somada às condições de vida atual do usuário?.

Assim, a escolha dos parâmetros para a estratificação de risco foi fundamentada, principalmente, na necessidade de definir o nível em que ocorrerá a assistência em saúde. Para tanto, os sinais e sintomas foram divididos em seis grupos, de acordo com a frequência em que se apresentam nas respectivas síndromes psicopatológicas, e foram pontuados de acordo com o nível de gravidade ${ }^{4}$.

Nas situações estratificadas como de baixo risco, como as que apresentam sintomas leves a moderados de depressão, ansiedade e somatização, os sintomas tendem a se sobrepor. Nestes casos, os cuidados devem ser oferecidos com intensidade progressiva e os grupos não devem ser direcionados nem divulgados apenas para pessoas portadoras de um diagnóstico. A estratégia recomendada é iniciar com cuidados de baixa intensidade (atividade física em grupo, panfletos de autoajuda, grupos de apoio), passando por grupos psicoeducacionais e de apoio que explorem questões como autoestima ou resiliência, evoluindo para o uso de terapia medicamentosa com supervisão especializada e psicoterapia em grupo ou individual, caso necessário'.

É importante lembrar que, na maioria das vezes, o enfermeiro é o coordenador da equipe da Estratégia Saúde da Família (ESF), e um dos grandes desafios para atender à saúde mental é o 
estabelecimento de sua competência 5 . Ele exerce um papel importante na assistência a pessoas com transtorno mental, como sensibilização da população sobre a importância de sua inserção na comunidade, inclusive colaborando e responsabilizando-se pela construção de novos espaços de reabilitação psicossocial, que farão com que esses indivíduos se sintam valorizados, afinal, a cidadania dessas pessoas e de sua família está assegurada na política de desinstitucionalização.

Os enfermeiros, portanto, precisam estar preparados para atender esses pacientes e suas famílias, sendo assim, a conduta adotada pelo profissional da ESF tem como objetivo apoiar e tratar a pessoa de modo a valorizar não apenas a doença, mas, principalmente, tratá-la de maneira integral, favorecendo a reinserção ao convívio social com medidas qualificadas 5 .

Diante do exposto, o estudo teve como objetivo conhecer a percepção do enfermeiro sobre o instrumento de estratificação de risco em saúde mental e quais ações de enfermagem são ofertadas ao paciente classificado como baixo risco.

\section{METODOLOGIA}

Estudo descritivo-exploratório, de abordagem qualitativa. A pesquisa exploratória visa ampliar, clarear e transformar conceitos e ideias, com o foco na elaboração de hipóteses que poderão ser pesquisadas em estudos seguintes ou na formulação de problemas mais precisos acerca de um tema. 0 estudo descritivo, por sua vez, tem o objetivo de descrever características de um grupo de pessoas ou situação ou então estabelecer relação entre variáveis?.

O estudo foi realizado nas Unidades Básicas de Saúde (UBSs) de um município do Noroeste do Paraná, que possui o selo ouro, prata e bronze de certificação ${ }^{8}$. Este projeto de qualificação das UBSs foi desenvolvido pela Secretaria do Estado de Saúde (SESA) em 2014 e já está presente em 312 municípios, beneficiando 902 postos de saúde. A iniciativa é conceder títulos de reconhecimento às equipes da atenção primária, com amplo processo de qualificação dos serviços básicos de saúde, o que inclui a assistência ofertada nas unidades de saúde. Por meio de uma metodologia baseada no conceito de tutoria, técnicos da SESA acompanham. orientam e prestam consultoria às equipes das unidades que aderem ao projeto ${ }^{8}$.

Sendo assim, a população do presente estudo englobou os enfermeiros das Equipes da ESF que atuam nas UBSs com o selo de qualidade. Atualmente, o município em estudo conta com duas equipes da ESF consideradas selo ouro e vinte e nove equipes classificadas como selo bronze. Devido a seis perdas por falta de funcionários, licença-maternidade e férias, a população de estudo contou com 25 enfermeiros da ESF.

Para coleta de dados foi realizada uma entrevista aberta com a seguinte questão norteadora: "Qual sua percepção sobre o instrumento de estratificação de risco em saúde mental e quais ações de enfermagem são ofertadas ao paciente classificado como baixo risco?" Além da questão norteadora, utilizaram-se questões de apoio e um questionário de identificação.

Para a realização das entrevistas, foi feito um pedido de autorização de pesquisa para a Secretaria Municipal de Saúde. Após a aprovação do estudo pela secretaria, foi realizado o primeiro contato com os enfermeiros. Neste primeiro contato foram explicados os objetivos do estudo. Após o aceite na participação, foram agendadas as entrevistas de acordo com a disponibilidade em relação ao melhor horário e data.

As entrevistas foram conduzidas pelo pesquisador principal e realizadas nas UBSs pertencentes a cada profissional entrevistado, em ambiente privativo, com duração média de 40 minutos. Foram finalizadas quando ocorreu a saturação dos dados qualitativos, ou seja, interrompeu-se a coleta de dados quando se constatou que elementos novos para subsidiar a 
teorização almejada não foram mais depreendidos a partir do campo de observação .

Os dados das entrevistas foram gravados em dispositivos eletrônicos de áudio, transcritos e analisados utilizando o método de análise de conteúdo Modalidade Temática de Bardin ${ }^{10}$. Este método de análise visa o desvendar crítico, primordialmente, através da descrição e de inferências que buscam esclarecer as causas de um problema ou as possiveis consequências advindas do mesmo. Esta análise está dividida em 1) Préanálise: engloba preparação do material, construção de hipóteses e formulação de indicadores que irão nortear a interpretação final; 2) Exploração do material: neste momento os dados são codificados, ou seja, transformados sistematicamente e agrupados em unidades de registro, que podem ser temas, palavras ou frases; 3) Tratamento dos resultados: trabalhos realizados através da inferência, que é guiada por vários pólos de atenção/ comunicação. Estes são esclarecidos e, em seguida, novos temas e dados são descobertos, fazendo-se necessária a comparação entre enunciados e ações, a fim de verificar possíveis unificações ${ }^{10}$.

A pesquisa foi desenvolvida de acordo com os trâmites determinados pela Resolução 466/2012 do Conselho Nacional de Saúde, com a assinatura do Termo de Consentimento Livre e Esclarecido (TCLE) e aprovação do Comitê Permanente de Ética em Pesquisa com Seres Humanos (COPEP) (CAAE 95234418.0.0000.0104). O sigilo e anonimato dos participantes foram preservados e as identificações foram feitas pela letra $E$ seguidas pelo número correspondente à inclusão da sua entrevista.

\section{RESULTADOS}

Foram entrevistados 25 enfermeiros da ESF. com predominância do sexo feminino (22 mulheres). idade entre 31 e 56 anos. Em relação ao tempo de atuação desses profissionais dentro da unidade, variam em torno de 6 meses a 14 anos. Quanto ao instrumento de estratificação de risco, 100\% dos entrevistados citaram que utilizam em sua rotina e, ao serem questionados sobre a dificuldade do instrumento, $100 \%$ dos profissionais referiram alguma dificuldade.

Dentre as falas obtidas foi possível elencar três categorias: "Ações em saúde mental realizadas pelos enfermeiros da ESF"; "Instrumento de estratificação de risco em saúde mental: potencialidades e fragilidades" e "Dificuldades das Equipes da ESF em saúde mental".

\section{AÇÕES EM SAÚDE MENTAL REALIZADAS PELOS ENFERMEIROS DA ESF}

Por meio dos relatos dos entrevistados foi possível observar a importância das ações desenvolvidas na UBS para os pacientes estratificados como baixo risco, sendo elas: grupos de escuta e acolhimento, grupos de convivência, atividades de leitura e exercícios físicos.

"O baixo risco a gente mantém no acompanhamento em grupo. [...] porque às vezes a pessoa acha que precisa de remédio, mas às vezes ela só precisa estar inserida em alguma atividade [...] terapia de grupo acaba com a fila de espera e resolve a maior parte dos problemas" (E13).

"[...] tem o grupo de convivência lá no salão. [...] com a orientação que ela [psicóloga] faz, ela consegue fazer com que eles tenham vontade de voltar, porque ali eles se sentem bem, acolhidos. [...] aí uma vez por mês eles têm uma confraternização, aí vai outro profissional para falar de um tema" (E7).

"A gente tem um grupo de mulheres de leitura, específico para saúde mental" (E17).

"Agora o médico já faz um mês que ele está com um grupo de saúde mental nas quintas à tarde. mas ele é aberto para a população independente e se faz uso de medicação, se é alto risco, baixo ou familiar" (E18) 
Porém foi possível observar que grande parte das UBSs intitula por "grupo de saúde mental" um grupo de pessoas agendadas para consulta médica, a fim de replicação de receitas medicamentosas.

"[...] o paciente de baixo risco a gente inclui no nosso grupo de saúde mental que chama azul turquesa, nesse grupo a médica da família acompanha esses pacientes com algum tipo de tratamento medicamentoso, em geral psicotrópicos, então a cada dois meses a gente atende esses pacientes nesse grupo. Em geral está sendo feito só o fornecimento de receitas" (E23).

"[...] não existe um grupo, a gente chama de grupo de saúde mental, mas é aquele dia dos pacientes que usam psicotrópicos, mas não é um grupo de atividade ou trabalho, poderia ser dia da consulta médica para receita controlada, é bem isso" (E4).

Além disso, as unidades contam com o apoio matricial do Núcleo de Apoio à Saúde da Família (NASF), profissionais fundamentais para a realização de algumas atividades.

"[...] nos grupos de atividade física a gente tem o apoio do NASF e às vezes no grupo de apoio com o psicólogo também do NASF [...]" (E1).

"[...] aqui tem o grupo primavera, é específico para saúde mental, é tocado pela equipe no NASF que trabalha abordando vários temas" (E13).

"[...] eu acho que com um matriciamento fica melhor, um apoio matricial para esses casos de baixo risco" (E14).

\section{INSTRUMENTO DE ESTRATIFICAÇÃO DE RISCO EM SAÚDE MENTAL: POTENCIALIDADES E FRAGILIDADES}

Em relação às potencialidades trazidas com o instrumento de estratificação de risco em saúde mental, alguns dos entrevistados o caracterizaram como facilitador da assistência, referindo ainda que as capacitações oferecidas pela Secretaria de Saúde foram úteis.

"No começo quando foi implantado a gente teve [dificuldade], mas depois que a gente teve a capacitação, foi útil e deu para aprender" (E14).

"A gente fez uma atualização há uns dois ou três meses atrás, eu tinha algumas dúvidas e consegui tirar [...] eu tenho dificuldade, mas melhorou depois do treinamento" (E7).

Além disso, foi citado que conhecer o instrumento e utilizá-lo com frequência auxilia na sua aplicação.

"[...] quando começa uma primeira conversa com o paciente, muitas vezes eu nem faço a pergunta, eu já vou conseguindo pontuar na própria conversa. Então se você entender bem e utilizar com frequência o instrumento, você quase nem precisa ficar perguntando item por item" (E13).

"[...] ele já está há alguns anos conosco [...] eu imprimo e sigo o check-list" (E5).

Sobre as fragilidades deste instrumento os entrevistados mencionaram a falta de tempo e a alta demanda como fatores negativos.

"[...] é muito pouco tempo para você conseguir pegar de um paciente que fica ali com você vinte minutos, meia hora, porque é um questionário amplo [...]" (E7)

"[...] o tempo que a gente tem para fazer isso é uma dificuldade, porque cada pergunta que você faz em forma de conversa, gera um monte de resposta" (E4).

Os termosutilizados paracompor oinstrumento foram caracterizados pelos profissionais como complexos e difíceis, se constituindo em fator de fragilidade na sua aplicação. 
"[...] geralmente eu não pergunto o que está escrito ali não, eu tento ler e interpretar e algumas coisas eu até não pergunto, porque é muito complicado, tinha que ser um negócio bem mais simples" (E21).

"[...] não tenho conhecimento desses nomes técnicos que são usados na psiquiatria, então eu acho que dificulta um pouco [...]" (E8)

"Tenho dificuldades. Nas crises convergentes e dissociativas [...] e muitas vezes eu confundo alucinação com delírio [...]" (E6).

Durante os depoimentos foi destacada a subjetividade do instrumento em virtude dos diferentes olhares dos profissionais que o aplicam.

"[...] enquanto enfermeira eu tenho um olhar e muitas vezes ou a maioria delas encaminha para o médico, aí ele tem outro olhar, a estratificação muda, quando chega no psiquiatra é o mesmo caso [...]" (E2O).

"[...] tem alguns termos que é complicado, quanto ao meu entendimento, quanto profissional de enfermagem, para a psicologia já é mais fácil [...]" (E16).

"[...] eu acho que é uma área mais voltada para psicologia do que para enfermagem" (E24).

\section{DIFICULDADES DAS EQUIPES DA ESF EM SAÚDE MENTAL}

O excesso de encaminhamentos para a especialidade em saúde mental, tanto por parte dos profissionais como pela solicitação dos pacientes para a psicologia e/ou psiquiatria, foi uma dificuldade relatada pela equipe da ESF, ocasionando aumento do número de consultas especializadas.

"[...] os que não tomam [medicação], se você avaliar e dá quase médio, a gente acaba pontuando médio para ter a questão do psiquiatra [...]" (E7).
"[...] às vezes ele [paciente] vem aqui querendo uma consulta com o psiquiatra, achando que todo mundo pode passar com o psiquiatra [...]" (E14).

"[...] tem muito médico na unidade que prefere passar a bola para frente [...]" (E8).

"[...] a nossa fila de psicologia na unidade é muito grande, o paciente demora muito tempo para ser acolhido pela psicologia e depois vai aguardar um outro tempo para ser feita a terapia [...]" (E3).

Além disso, a falta de tempo para realizar uma escuta qualificada foi evidenciada como uma das dificuldades encontradas pelas equipes de ESF, assim como a elevada demanda de atendimento, sendo esses dois fatores totalmente relacionados.

"[...] se eu ficar uma hora escutando, eu tenho uma fila enorme atrás daquela pessoa me esperando para atender" (E2).

"O problema nosso é a sobrecarga e a demanda muito grande, porque esse paciente precisa de um cuidado maior" (E19)

Em relação ao acompanhamento dos pacientes após a estratificação, observou-se a falta de continuidade da atenção, acarretando problemas como falta de adesão dos pacientes e resistência dos mesmos.

"[...] os de baixo risco não dão trabalho. É só buscando a receitinha" (E3).

"Só quando tem sofrimento mental é que eles são inseridos em um grupo de saúde mental que tem aqui, então só médio e alto risco, porque não tem suporte para absorver e mesmo se a gente encaminhasse eles não viriam [...]" (E16).

"[...] são pouco aderentes, tomam um pouco o remédio e logo desaparecem, depois voltam piores. [...] eles ficam muito bravos porque é falado que só vai passar com o médico clínico geral, aí falam que você está fazendo pouco caso" (E14). 
"[...] por eles, eles só viriam e pegariam a receita na recepção" (E4).

Outra fragilidade relatada pelas equipes foi a falta de afinidade em trabalharem com a saúde mental, sendo necessário investir em treinamentos e capacitações.

"[...] desde estudante, da graduação, uma das áreas que eu não me identificava era a saúde mental [...] eu acho muito complexo lidar com pessoas da saúde mental" (E2)

"[...] faço tudo que o protocolo pede, só que não sou a melhor pessoa para trabalhar com pessoas com problemas de depressão, ansiedade, problemas na saúde mental [...] não é que eu tenha dificuldade, é que eu não gosto [...] às vezes eu acabo não ajudando o quanto que o paciente precisaria" (E15)

"[...] os de baixo risco podem virar médio ou alto risco e você não sabe o que fazer [...]" (E7).

\section{DISCUSSÃO}

Em vista dos resultados obtidos foi possível observar a importância das atividades ofertadas pelas UBSs para este grupo de pacientes classificados como baixo risco em saúde mental pelo instrumento proposto pela SESA, como, por exemplo, os grupos de convivência, de atividades de leitura e atividades físicas.

No entanto, quando se fala em educação em saúde, percebe-se a escassez de ações ofertadas no âmbito da atenção primária específica para esta clientela. Estes dados estão de acordo com estudo realizado com 134 enfermeiros atuantes na ESF em Terezina (PI), demonstrando que somente $5 \%$ das atividades voltadas à saúde mental são de terapia comunitária. Este aspecto permite observar que, na ESF, os profissionais comumente não realizam atividades voltadas especificamente à
Saúde Mental11․ Neste sentido, nota-se a fragilidade apresentada pelos enfermeiros em identificar e operacionalizar ações que atendam a saúde das pessoas em sofrimento psíquico como parte inclusiva do processo de trabalho na Saúde da Família².

Em alguns aspectos relacionados a esta dificuldade de realizar atividades em saúde mental estão a baixa qualificação das equipes, sentimentos de angústia diante da complexidade das situações, abordagens clínicas tradicionais (queixa-conduta) somadas à complexidade das questões relacionadas à vulnerabilidade social e as altas prevalências de problemas de saúde mental. Este panorama sugere que profissionais e gestores públicos intensifiquem a criação de novas estratégias de formação e apoio continuado à Atenção Primária12.

Um outro estudo realizado na atenção primária com os profissionais de saúde aponta que a principal dificuldade relatada foi a falta de profissionais especializados para realizar o cuidado em saúde mental ${ }^{13}$. Neste sentido, e visando sanar essas dificuldades, a educação permanente precisa ser vista como um processo dinâmico e contínuo de construção do conhecimento, pautado no diálogo, em que todos os atores assumam papel ativo no processo de aprendizagem, por meio de uma abordagem crítica reflexiva da realidade, o que reafirma a ideia do Paradigma Psicossocial14.

Para sanar as dificuldades referidas, algumas equipes de saúde contam com o apoio matricial do NASF, que se configura como um suporte técnico especializado, que é ofertado a uma equipe interdisciplinar de saúde a fim de ampliar seu campo de atuação e qualificar suas ações. Ele pode ser realizado por profissionais de diversas áreas especializadas $^{15}$. 0 apoio matricial representa um arranjo organizacional na atenção básica, visando a ampliação e reorientação da demanda para a saúde mental.

Fundamentado no princípio da responsabilização compartilhada, ele pretende produzir maior resolutividade à atenção em saúde². 
Então, a partir de discussões clínicas conjuntas com as equipes ou mesmo intervenções conjuntas concretas (consultas, visitas domiciliares, entre outras), os profissionais de Saúde Mental podem contribuir para o aumento da capacidade resolutiva das equipes, qualificando-as para uma atenção ampliada em saúde que contemple a totalidade da vida dos sujeitos.

O apoio matricial elimina a lógica de encaminhamentos para outros serviços e elabora projetos terapêuticos que podem ser desenvolvidos na atenção básica². Neste sentido, o Apoio Matricial se torna uma forma de compartilhar responsabilidades. Ele exige que os profissionais, juntos, compreendam o caso e suas necessidades, para só então verificar os encaminhamentos indiscriminados e decidir sobre as responsabilidades de cada profissional ${ }^{16}$.

Com isso, é possível evitar práticas que levam à psiquiatrização e à medicalização do sofrimento e, ao mesmo tempo, promover a equidade e o acesso, garantindo um atendimento direcionado às vulnerabilidades e potencialidades de cada usuário ${ }^{16}$.

Um estudo realizado em Botucatu (SP)15 apontou que a maior parte da demanda em Saúde Mental são casos considerados leves. Assim, evidenciou-se que o Apoio Matricial pode ajudar a aumentar a capacidade resolutiva das equipes de referência, para que os profissionais de Saúde Mental possam abrir sua agenda para os casos graves. Para isso, considerou-se necessário adequar a clínica da Saúde Mental às necessidades da população e do contexto da saúde coletiva. Em seguida foram listados recursos a serem usados como alternativas à assistência tradicional da saúde mental, no sentido da ampliação da clínica tanto das equipes de referência quanto da própria saúde mental, como grupos de convivência, espaço cultural, oficinas e outros ${ }^{15}$.

Para direcionar o fluxo dos atendimentos em saúde mental a fim de garantir uma assistência de maior resolutividade, no Estado do Paraná está sendo preconizado o uso do instrumento de estratificação de risco em saúde mental'. Neste sentido, a SESA recomenda que a estratificação de risco deva ser realizada considerando a gravidade dos sinais e sintomas apresentados, sem necessidade de firmar diagnóstico inicial, e somada às condições de vida atual do usuário.

Deve-se atentar que os transtornos mentais, por sua característica de cronicidade, tendem a oscilar em sua necessidade de atenção ao longo da vida, trazendo dúvidas entre os profissionais de enfermagem devido à subjetividade do tratamento, por se tratar de agravos complexos e multifatoriais, e que para definir sua gravidade ou risco faz-se necessário considerar a presença de outros fatores considerados agravantes e atenuantes?.

Neste sentido os profissionais do estudo sentiram-se na maioria das vezes inseguros em utilizar o instrumento de estratificação em virtude da pouca familiaridade com os termos técnicos que o constituem, pelo tempo gasto em sua aplicação, e pela subjetividade do instrumento, o que pode ocasionar uma pontuação supervalorizada e encaminhamentos desnecessários ao especialista. Neste sentindo torna-se importante minimizar as inferências pessoais e se concentrar no histórico da pessoa ${ }^{17}$.

Em pesquisa realizada com enfermeiros da ESF do município de São Gonçalo (RJ)² demonstrou-se que os enfermeiros apresentam dificuldades em identificar sofrimento psíquico no âmbito da ESF. Ao se depararem com uma pessoa em sofrimento, imediatamente encaminham-no aos profissionais especialistas. Não demonstrando assim, disponibilidade para o acolhimento, escuta e apoio social dessas pessoas. Além disso, os enfermeiros referem que a equipe não tem suporte para oferecer aos pacientes encaminhando os casos aos psiquiatras e/ou psicólogos².

Ainda que, de uma forma geral, haja certa abertura para o tratamento de pacientes com problemas psicossociais, ou sofrimento mental mais leve, tais como depressões e ansiedades, alguns profissionais médicos afirmam se sentir à vontade 
apenas para prescrever antidepressivos, mas não ansiolíticos, e muito menos antipsicóticos. Isso revela a dificuldade sentida por estes profissionais na condução do sofrimento mental, fazendo com que haja aumento da quantidade de encaminhamentos para a especialidade ${ }^{13}$.

A fragmentação do atendimento torna-se uma fragilidade recorrente nos atendimentos aos pacientes em sofrimento mental. Um exemplo disto são os casos identificados como sendo de saúde mental, mas que não pertenciam a nenhum serviço de referência. Apesar da existência da Rede de Atenção Psicossocial, ainda existe pouca integração entre os diversos pontos de atenção e a atenção primária. O vínculo frágil entre os serviços é atribuído tanto à alta rotatividade de profissionais como também aos modelos de assistência que sofrem alterações a cada eleição municipal, o que atrapalha a continuidade da proposta ${ }^{13}$.

Sobre o acompanhamento dos pacientes após a estratificação de risco, observou-se uma carência de seguimento efetivo por parte da equipe de saúde. Observa-se que os pacientes, em sua grande maioria, comparecem ao serviço no atendimento em grupo apenas para a troca de receita e retirada da medicação prescrita pelo médico. Esse modelo é visto pela equipe como positivo, visto que os pacientes de baixo risco, na percepção dos profissionais, não exigem um nível complexo de tratamento, sendo resolvido apenas com a prescrição de medicação.

A ausência de seguimento clínico e a falta de avaliações periódicas dos pacientes que usam psicotrópicos criam práticas de repetição de receitas. Além disso, em estudo realizado ${ }^{12}$, muitos pacientes reconhecem a existência de outros tipos de tratamentos como o atendimento psicológico, e/ou Centro de Convivência, mas queixam-se que formas de tratamentos alternativos não são propagadas pelas equipes quanto gostariam e referiram que eles auxiliariam muito mais do que o próprio remédio.

Sendo assim, a ausência de um planejamento integrado das ações voltadas para a atenção em saúde mental na unidade indica um cuidado não integral das questões de saúde, em que o usuário em sofrimento psíquico aparece como um elemento estranho e desestabilizador na rotina assistencia dos profissionais. Portanto, a incorporação do acolhimento e do vínculo no cotidiano do cuidado em saúde deve ser implantada, pois ela também tem contribuído para problematizar a qualidade dos atendimentos ${ }^{13}$.

Sabe-se que a saúde mental é considerada uma área complexa, como pode ser constatado nos depoimentos dos entrevistados, tanto pela falta de afinidade com a temática quanto pela sua complexidade. Em concordância com esse fato, um estudo realizado em unidades de saúde de dois municípios do Estado do Ceará sugere a falta de preparo dos profissionais de saúde da rede básica para receber e cuidar de pessoas com transtornos psíquicos, principalmente quando os usuários são pessoas com histórias de múltiplas internações psiquiátricas $^{18}$

Outro aspecto importante referido é a discriminação que se observa por parte de profissionais em relação ao cuidado da pessoa com transtorno mental. Aponta-se que essa discriminação pode se manifestar sob a forma de recusa transpassando o nível psíquico e atingindo o aspecto físico deste indivíduo. Ainda são muito frequentes as atitudes moralizantes e repressivas quando não se sabe lidar com a situação, ou quando não se dispõe de ações comunitárias para enfrentar, ou pelo menos refletir sobre problemas como o alcoolismo, as reações exacerbadas de medo entre outras ${ }^{14}$.

O problema maior a ser enfrentado é que a maioria desses profissionais se sente despreparada para o manejo das pessoas com transtornos mentais, o que gera sentimento de impotência e frustração. Portanto, as instituições de ensino e outras devem proporcionar uma formação adequada aos profissionais que terão o papel cada vez maior no cuidado a essas pessoas. Isso não implica exigir 
dos mesmos a substituição dos especialistas, tendo em conta a sua finalidade de ação universalista e generalista, mas em muni-los com recursos capazes de torná-los reais colaboradores em seu nível de complexidade ${ }^{13}$.

Neste sentido, a atenção primária por ser o primeiro acesso do usuário a um serviço de saúde deve investir no vínculo e acolhimento. A ausência de tempo para uma escuta qualificada relacionada à alta demanda de usuários foi uma dificuldade enfrentada pelos entrevistados, que evidenciaram alta demanda em saúde mental, que são geradoras de dificuldades de articulação em uma rede de cuidados.

Neste sentido, as equipes sobrecarregadas têm dificuldade de dar continuidade aos fluxos da rede. Além disso, a demanda de usuários na atenção primária produz angústia nas equipes e exige um trabalho de compreensão, o que tenciona certo tipo de procedimento mecanizado de queixaconduta $^{12}$. Além disso, a ausência de tempo para a escuta qualificada se torna uma fragilidade para a continuidade do cuidado, assim como a alta demanda de atendimentos ${ }^{18}$.

Pensando em um acompanhamento dos pacientes em saúde mental de qualidade e as formas do relacionamento com a clientela, os atendimentos deveriam priorizar o diálogo com a população, ampliando a escuta para o território, saindo das linhas de ação apenas empreendidas pelos trabalhadores especializados superando a visão terapêutica voltada apenas para a remissão dos sintomas psiquiátricos ${ }^{16}$.

\section{CONSIDERAÇÕES FINAIS}

É possivel compreender que as ações de enfermagem na Atenção Básica para os pacientes classificados como baixo risco em saúde mental são importantes, pois além de possuírem um caráter resolutivo das equipes em relação ao paciente, as qualificam para uma atenção ampliada em saúde que comtemple a totalidade da vida dos sujeitos.

Além disso, para ofertar essas ações com frequência e qualidade, as unidades contam com um apoio matricial do NASF, que elimina a lógica de encaminhamentos para outros serviços e elabora projetos terapêuticos que podem ser desenvolvidos na atenção básica. Neste sentido, o apoio matricial se torna uma forma de compartilhar responsabilidades.

A estratificação de risco, como foi apresentada, deve ser realizada considerando a gravidade dos sinais e sintomas apresentados. Porém os transtornos mentais tendem a oscilar em sua necessidade de atenção ao longo da vida, trazendo dúvidas entre os profissionais de enfermagem devido à subjetividade do instrumento.

Este fato sugere que gestores intensifiquem a criação de novas estratégias de formação e apoio continuado para os profissionais da Atenção Primária, para que sejam sanadas as dúvidas quanto à aplicação do instrumento de estratificação e quanto ao acompanhamento dos pacientes estratificados. Além disso, é necessário seguir o fluxograma de atendimento proposto pelo município para o encaminhamento adequado dos pacientes aos pontos de atenção da Rede Psicossocial.

Muitos profissionais se sentem despreparados para o manejo das pessoas com transtornos mentais, o que gera sentimento de impotência e frustração. Portanto as instituições de ensino têm papel primordial na formação dos futuros profissionais para que sejam capazes de prestar um cuidado de qualidade às pessoas com transtornos mentais.

Por fim, espera-se que esta pesquisa tenha contribuído no sentido de refletir sobre a estratificação de risco e as ações de saúde mental ofertadas pela Atenção Básica aos casos de baixo risco, almejando melhorar a qualidade do cuidado ofertado pela equipe multiprofissional. 


\section{REFERÊNCIAS}

1. Secretaria de Estado da Saúde do Paraná (SESA). Oficinas do APSUS: a rede de atenção à saúde mental no Paraná e a competência da APS. Curitiba: SESA; 2014

2. Amarante AL, Lepre AS, Gomes JLD, Pereira AV, Dutra VFD. As estratégias dos enfermeiros para o cuidado em saúde mental no Programa Saúde da Família. Texto contexto - enferm. 2011:20(1):85-93.

3. Organização Mundial de Saúde, Organização Mundial de Médicos de Família (Wonca). Integração da saúde mental nos cuidados de saúde primários: uma perspectiva global. Coordenação Nacional para a Saúde Mental. Portugal, 2008.

4. World Health Organization. Mental health policy, plans and programmes [Internet]. Geneva $(\mathrm{CH})$ : World Health Organization; 2004 [citado 2018 Set 3]. 98 p. Disponivel em: https://www.who. int/mental_health/policy/en/policy_plans_revision.pdf

5. Neves HG, Lucchese R, Munari DB. Saúde mental na atenção primária: necessária constituição de competências. Rev bras enferm. 2010;63(4):666-70.

6. Waidman MAP, Marcon SS, Pandini A, Bessa JB, Paiano M. Assistência de enfermagem às pessoas com transtornos mentais e às famílias na Atenção Básica. Acta Paul Enferm. 2012:25(3):346-51.

7. Gil AC. Métodos e técnicas de pesquisa social. 6 ed. São Paulo: Atlas; 2008.

8. Portal Saúde Maringá. Secretaria Municipal de Saúde. UBSs de Maringá recebem Selo Bronze e Ouro de Qualidade. Prefeitura Municipal de Maringá [Internet]. 2017 Set 23 [citado Set 3]. Disponivel em.: http://www2.maringa.pr.gov.br/site/index.php?sessao =b9eaadd9e455b9\&id=32137.

9. Fontanella BJB, Luchesi BM, Saidel MGB, Ricas J, Turato ER, Melo DG. Amostragem em pesquisas qualitativas: proposta de procedimentos para constatar saturação teórica. Cad Saúde Pública. 2011;27(2):388-94.

10. Bardin L. Análise de Conteúdo. São Paulo: Edições 70; 2011. 229 p.

11. Souza LGS, Menandro MCS, Couto LLM, Schimith PB, Lima RP. Saúde mental na Estratégia Saúde da Família: revisão de literatura brasileira. Saúde Soc. 2012:21(4):1022-34

12. Campos RO, Gama CA, Ferrer AL, Santos DVD. Stefanello S, Trapé TL et al. Saúde mental na atenção primária à saúde: estudo avaliativo em uma grande cidade brasileira. Ciênc saúde coletiva. 2011;16(12):4643-52.

13. Barbosa VFB, Cavalcanti A, Alcântara MCA, Pedroza RM, Ferreira SHV et al.. O papel da atenção primaria de saúde na construção das redes de cuidado em saúde mental. Rev Fund Care. 2017:9(3):659-68.

14. Almeida AB, Nascimento ERP, Rodrigues J, Schweitzer G. Intervenções nas situações de crise psíquica, dificuldades e sugestões de uma equipe pré-hospitalar. Rev bras enferm. 2014;67(5):70814.

15. Bonfim IG, Bastos ENE, Gois CWL, Tofoli LF. Apoio matricial em saúde mental: uma análise da produção científica e documental. Interface (Botucatu). 2013:17(45):287-300.
16. Quinderé PHD, Jorge MSB, Nogueira MSL, Costa LFA, Vasconcelos MGF. Acessibilidade e resolubilidade da assistência em saúde mental: a experiência do apoio matricial. Ciênc saúde coletiva. 2013:18(7):2157-66

17. Organização Mundial da Saúde, coordenador. Classificação cstatística internacional de doenças e problemas relacionados à saúde. São Paulo: Edusp: 2012. 1056 p.

18. Pinto AGA, Jorge MSB, Vasconcelos MGF, Sampaio JJC, Lima GP. Bastos VC et al. Apoio matricial como dispositivo do cuidado em saúde mental na atenção primária: olhares múltiplos e dispositivos para resolubilidade. Ciênc saúde coletiva. 2012:17(3):653-60.
RECEBIDO: 13/03/2019

ACEITO: 13/05/2019 